Cerebrovasc Dis 2008;25:281-282

DOI: $10.1159 / 000119637$

\section{Hypoglycemia-Induced Pontine Infarction in a Diabetic Male with Basilar Artery Stenosis: Insight into the Mechanisms of Hypoglycemic Stroke}

\author{
Siva P. Sontineni, Jennifer M. Lee, Joann Porter \\ Department of Medicine, Creighton University, \\ Omaha, Nebr., USA
}

Although hypoglycemic hemiplegia has long been recognized, it is only recently that the physiological alterations of cerebral blood flow (CBF) have been understood [1]. We report a patient who experienced a hypoglycemic episode and developed a permanent right-sided motor deficit. Subsequent MRI/MRA demonstrated acute pontine infarct and vertebro-basilar artery stenosis.

\section{Case Report}

A 60 -year-old black man was admitted with fever, productive cough, rhinorrhea and dyspnea. He had a history of hypertension and diabetes mellitus diagnosed 10 years ago as well as complications of hypertensive heart disease, diabetic retinopathy and erectile dysfunction. At admission he was febrile $\left(38^{\circ} \mathrm{C}\right)$, regular in his pulse ( 96 beats/min) with brachial cuff pressure of 198/108 $\mathrm{mm} \mathrm{Hg}$. His neurological examination was unremarkable except for very distal sensory impairment. A diagnosis of respiratory tract infection was made and treated with azithromycin. He also received furosemide in addition to lisinopril and nitroglycerine, which reduced his blood pressure to $156 / 72 \mathrm{~mm} \mathrm{Hg}$. Glipizide and metformin were continued and capillary glucose measurements ranged between 87 and $140 \mathrm{mg} / \mathrm{dl}$.

On the second day, he became obtunded with a new right hemiplegia, slurred speech and a National Institute of Health Stroke Scale score of 13. Bedside fingerstick glucose obtained was $42 \mathrm{mg} / \mathrm{dl}$ and the blood pressure was 154/82 $\mathrm{mm} \mathrm{Hg}$. One ampule of $50 \%$ dextrose was given intravenously. Noncontrast CT obtained immediately revealed no acute abnormalities. One hour later, his National Institute of Health Stroke Scale score was 6. Over the next $48 \mathrm{~h}$, the patient experienced little improvement of the residual right-sided motor deficit. On the third day MRI/ MRA demonstrated an area involving the left half of the pons and lower mid-brain showing $\mathrm{T}_{2}$ prolongation and restricted diffusion consistent with acute infarct. Along with this new pontine infarct (fig. 1), 50\% mid-basilar artery stenosis (fig. 2) was also demonstrated on the MRA. Five days later, he was discharged to a rehabilitation facility.

\section{Discussion}

Hypoglycemia is known to cause neurologic deficits including hemiparesis or hemiplegia. The pathogenetic mechanisms proposed include: acute brain injury [2-5], selective neuronal vulnerability [6-8], cerebral vasospasm [9] and asymmetric CBF [10]. Studies indicate that the CBF increases in response to hypoglycemia $[1,10]$. The relative distribution of CBF during hypoglycemia is altered by unknown mechanisms. When ten regions of the brain were analyzed in healthy men during insulin-induced hypoglycemia, the highest increments of $\mathrm{CBF}$ were found in frontal and parietal lobes, and the lowest in the pons/brain stem regions [1]. Similar measurements of global cerebrovascular resistance showed an overall decrease [10]. In the presence of cerebrovascular stenosis, the overall increased flow to the non-stenotic areas can lead to clinically significant steal phenomenon as evident in our patient. This is particularly important in the brain stem area

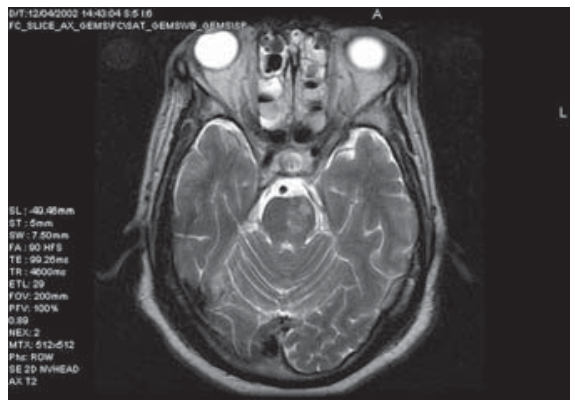

Fig. 1. An area involving the left half of the pons (in the picture) and lower mid-brain (not shown here) that shows $\mathrm{T}_{2}$ prolongation and restricted diffusion consistent with acute infarct.

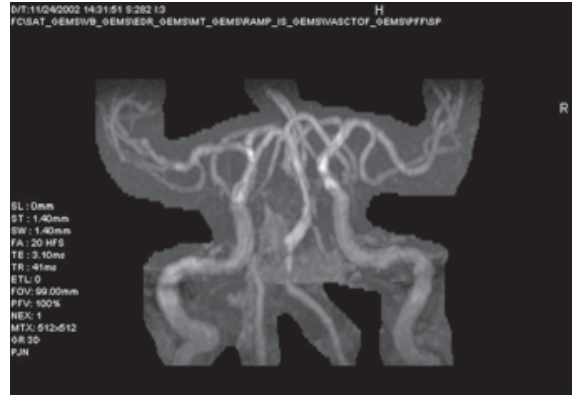

Fig. 2. MRA. Within the basilar artery, there are two areas of narrowing, with mild narrowing in the proximal-basilar artery and approximately $50 \%$ narrowing in the mid-basilar artery.

\begin{tabular}{ll}
\hline KARGER & @ 2008 S. Karger AG, Basel \\
1015-9770/08/0253-0281\$24.50/0 \\
$\begin{array}{l}\text { E-Mail karger@karger.ch } \\
\text { www.karger.com }\end{array}$ & $\begin{array}{l}\text { Accessible online at: } \\
\text { www.karger.com/ced }\end{array}$
\end{tabular}


due to the redistributory changes of blood flow in face of hypoglycemia. Earlier reports indicate that hypoglycemia in the absence of cerebrovascular stenosis can cause reversible injury of internal capsule and splenium, demonstrated on MRI in a patient with transient hypoglycemic hemiparesis [11]. Kim et al. [11] reported complete resolution of the lesions within $12 \mathrm{~h}$ after appropriate correction of hypoglycemia, as did neurological deficits, indicating that the lesions are reversible cytotoxic edema in nature. However, in the current case the lesions were demonstrable on the third day after the onset of hemiparesis, refuting the possibility of reversible cytotoxic edema. Lacunar pontine strokes can present in a similar fashion; however, the temporal relation between the onset of hypoglycemia, neurologic deficit and partial resolution of deficit with dextrose infusion in this patient clearly establishes the cause and effect relationship.

We propose that the presence of basilar stenosis made the patient vulnerable to pontine infarct in the setting of hypoglycemiainduced cerebral flow patterns. This is the first reported case of such hypoglycemic brain injury resulting in pontine infarct in a patient with diabetes and basilar artery stenosis. Underlying cerebrovascular disease is a frequent occurrence in the elderly, diabetic population. Knowledge of vertebro-basilar stenosis maylead the physician to more fastidious management of blood glucose.

\section{References}

1 Tallroth G, Ryding E, Agardh CD: Regional cerebral blood flow in normal man during insulin-induced hypoglycemia and in the recovery period following glucose infusion. Metabolism1992;41:717-721.

2 Wattoo MA, Liu HH: Alternating transient dense hemiplegia due to episodes of hypoglycemia. West J Med 1999;170:170-171.

3 Vanpee D, Donckier J, Gillet JB: Hemiplegia hypoglycemia syndrome. Eur J Emerg Med 1999;6:157-159.

4 Bolyn-Starks L: Hypoglycemic hemiplegia: a case study. Heart Lung 1995;24:330-332

5 Shirayama H, Ohshiro Y, Kinjo Y: Acute brain injury in hypoglycaemia-induced hemiplegia. Diabetes Med 2004;21:623-624.

6 Mazziotta JC, Phelps ME, Carson RE, Kuhl DE: Tomographic mapping of human cerebral metabolism: sensory deprivation. Ann Neurol 1982 12:435-444.

7 Hypoglycemia and the nervous system. Lancet 1985;ii:759-760.

8 Shintani S, Tsuruoka S, Shiigai T: Hypoglycemic hemiplegia: a repeat SPECT study. J Neurol Neurosurg Psychiatry 1993;56:700-701.

9 MacDonald JT, Brown DR: Acute hemiparesis in juvenile insulin-dependent diabetes mellitus (JIDDM). Neurology 1979;29:893-896.

10 Jarjour IT, Ryan CM, Becker DJ: Regional cerebral blood flow during hypoglycemia in children with IDDM. Diabetologia 1995;38:10901095.

$11 \mathrm{Kim} \mathrm{JH}, \mathrm{Roh} \mathrm{JH}, \mathrm{Koh} \mathrm{SB}$ : Reversible injury of internal capsule and splenium in a patient with transient hypoglycemic hemiparesis. Cerebrovasc Dis 2006;22:282-283.

Siva P. Sontineni, MD

Department of Internal Medicine

Creighton University Medical Centre

$601 \mathrm{~N}$ 30th St. Suite 5850

Omaha, NE 68131 (USA)

Tel. +1 402280 4566, Fax +1 4022804220

E-Mail ssontineni@gmail.com
Cerebrovasc Dis 2008;25:282-283

DOI: $10.1159 / 000119638$

\section{Brain Haemorrhage and Cerebral Vasospasm Associated with Chronic Use of Cannabis and Buprenorphine}

\author{
Dimitri Renard, Nicolas Gaillard \\ Department of Neurology, CHU Montpellier, \\ Hôpital Gui de Chauliac, Montpellier, France
}

A 34-year-old woman presented with subacute-onset headache. Her medication consisted of oral buprenorphine $5 \mathrm{mg}$ o.d. in treatment for heroin addiction, which she had stopped using 8 years before. No recent change in the dose of buprenorphine was made. She had smoked 2-6 cannabis cigarettes per day since the age of 22 . The day of symptom onset, she smoked 4 cannabis cigarettes followed by another one $50 \mathrm{~min}$ after headache onset. No alcohol or other drugs were used. Except for tobacco, no other vascular risk factors were present. Clinical examination was normal. The blood pressure was 145/86 $\mathrm{mm} \mathrm{Hg}$ on admission and normalized spontaneously $1 \mathrm{~h}$ later. Blood count, CRP, sedimentation rate, renal and liver function tests, ANF, lupus anticoagulant, anticardiolipin antibodies, ANCA, and serology of HIV, syphilis, hepatitis $\mathrm{B}$ and $\mathrm{C}$ were normal. Urine toxicological screening was negative for cocaine, heroin and amphetamines, and strongly positive for cannabis. Unenhanced brain CT showed a right temporal lobe haemorrhage (fig. 1) without enhancement after contrast administration. No underlying acute brain infarction was seen on diffusion-weighted MRI. Transoesophageal echocardiography was normal. Angiography revealed diffuse multifocal arterial narrowing (fig. 1) in the absence of aneurysm or arteriovenous malformation. The buprenorphine dose was decreased to $2 \mathrm{mg}$ o.d. and the cannabis use was reduced to 3-4 cigarettes per week. Three months later, complete disappearance of arterial abnormalities was seen on angiography.

\section{Discussion}

Drug abuse is a risk factor for both haemorrhagic and ischaemic stroke [1]. Haemorrhagic stroke is most often seen with sympathomimetic drugs (e.g. cocaine, amphetamine and ephedrine) $[2,3]$. Possible aetiological mechanisms include acute hypertension, failure of cerebrovascular autoregulation, underlying aneurysm or arteriovenous malformation, coagulopathy, vasculitis and early haemorrhagic transformation of infarction. Cerebral vasoconstriction has been described in association with cocaine, amphetamines, ephedrine, phenylephrine, LSD and heroin $[2,4-$ 8]. Drug-induced vasculitis is often difficult to distinguish from vasospasm caused by these agents. Arterial narrowing disappearing after decrease in drug dose, like in our patient, favours vasospasm over vasculitis. Brain haemorrhage and cerebral vasoconstriction have never been described in association with cannabis or buprenorphine. In our patient, both drugs were well tolerated for a long period of time. One might question if there is a causal relationship between radiological abnormalities and these drugs. It is likely that vasoconstriction was related to (at least one of) these drugs, since angiographic abnormalities disappeared after decrease in the dose of both drugs. The pathophysiological role of 